Research Paper

\title{
The Correlation Between PARPI and BRCA1 in AR Positive Triple-negative Breast Cancer
}

\author{
Jiayan Luo*, Juan Jin*, Fang Yang, Zijia Sun, Wenwen Zhang, Yaqin Shi, Jing Xu and Xiaoxiang Guan ${ }^{凶}$ \\ Department of Medical Oncology, Jinling Hospital, Medical School of Nanjing University, Nanjing 210002, China. \\ * These authors contributed equally to this study. \\ $\square$ Corresponding author: Professor Xiaoxiang Guan, Department of Medical Oncology, Jinling Hospital, Medical School of Nanjing University, 305 East \\ Zhongshan Road, Nanjing, Jiangsu Province, 210002, P. R. China. Email: xguan@nju.edu.cn.
}

() Ivyspring International Publisher. Reproduction is permitted for personal, noncommercial use, provided that the article is in whole, unmodified, and properly cited. See http://ivyspring.com/terms for terms and conditions.

Received: 2016.05.16; Accepted: 2016.07.21; Published: 2016.11.25

\begin{abstract}
Triple-negative breast cancer (TNBC) lacks estrogen receptor (ER), progesterone receptor (PR) and human epidermal growth factor receptor 2 (HER-2) expression and thus cannot benefit from conventional hormonal or anti-HER2 targeted therapies. Anti-androgen therapy has shown a certain effect on androgen receptor (AR) positive TNBC. The emerging researches have proved that poly (ADP-ribose) polymerase (PARP) inhibitor is effective in BRCAl-deficient breast cancers. We demonstrated that combination of AR antagonist (bicalutamide) and PARP inhibitor (ABT-888) could inhibit cell viability and induce cell apoptosis significantly whatever in vitro or in vivo setting in AR-positive TNBC. Previous studies have proved that both BRCAI and PARPI have close connections with $A R$ in prostate cancer. We explored the correlation among AR, PARPI and $B R C A 1$ in TNBC for the first time. After BRCAI overexpression, the expression of AR and PARPI were decreased in mRNA and protein levels. Additionally, AR positively regulated PARPI while PARPI also up-regulated AR expression in vitro. We also confirmed BRCAl expression was negatively correlated with AR and PARP1 in TNBC patients using a tissue microarray with TNBC patient samples. These results suggest that the combination of bicalutamide and PARP inhibitor may be a potential strategy for TNBC patients and merits further evaluation.
\end{abstract}

Key words: AR, PARP1, BRCA1, combination therapy, triple-negative breast cancer.

\section{Introduction}

Triple-negative breast cancer (TNBC) is a highly aggressive breast cancer subtype, and defined by lack of estrogen receptor (ER) and progesterone receptor (PR) expression as well as amplification of human epidermal growth factor receptor 2 (HER-2) expression. TNBC accounts for approximately $15 \%$ of all breast cancers and occurs more frequently in young women and BRCA1 mutation carriers [1]. The treatment of TNBC has been challenging since it is defined due to no benefit from hormonal therapy or therapies targeting HER-2[2]. Currently, apart from surgery and chemotherapy, there are no effective targeted therapies for the disease. Searching for more therapeutic targets for TNBC patients is the primary aim of many studies.
Androgen receptor (AR) is a member of the steroid receptor superfamily which is expressed in more than $70 \%$ of breast cancer and in about $15 \%$ of TNBC $[3,4,5]$. Positive expression of AR in TNBC patients was reported to be associated with worse clinical outcomes, indicating that AR blockade might be a potential endocrine therapy for TNBC [6]. Bicalutamide, as a non-steroidal androgen receptor antagonist, can bind to AR and inhibit its activation [7]. A phase II study that bicalutamide was at a dose of $150 \mathrm{mg}$ daily in women with metastatic AR-positive TNBC obtained an endpoint of clinical benefit rate of $19 \% \quad(95 \% \quad \mathrm{CI}, 6 \%$ to $37 \%)$ and a median progression-free survival (PFS) of 12 weeks (95\% CI, 11 to 22 weeks) (NCT00468715), suggesting AR 
antagonist could be a treatment option for AR-positive TNBC patients [8]. Other clinical trails of anti-androgen bicalutamide in AR-positive TNBC patients are ongoing (NCT02353988) (NCT02348281). Lehmann et al. have classified TNBC into six subtypes based on their gene expression profiles. Among these subtypes of TNBC, gene expression in LAR subtype is most differential because it is highly enriched in hormonally regulated pathways though ER and PR are negative in this subtype. Further investigation finds that tumors in LAR group express high levels of $\mathrm{AR}$ and numerous downstream AR target factors [1]. According to these, AR may be possibly an important prognostic marker and therapeutic target of TNBC.

Poly (ADP-ribose) polymerase 1 (PARP1), the most abundant protein of the PARP superfamily, is involved in many cellular signal pathways including DNA replication, transcription, chromatin remodeling, telomere integrity, cell survival and death [9-14]. In DNA damage repair (DDR) process, PARP1 plays a curial role in the base-excision repair (BER) pathway for DNA single-strand breaks repair while DNA double-strand breaks are mainly repaired by homologous recombination repair (HRR) pathway in which BRCA1 has an important role [15-17]. As HRR pathway is impaired in BRCA1-mutated tumor cells, PARP inhibition in these cells can lead to accumulation of DNA damage and ultimately induce cell death because of impaired DDR from both BER and HRR dysfunctions [18]. TNBCs share many pathologic and clinical characteristics with BRCA-mutated breast cancers [19], which provides a rationale for clinical use of PARP inhibitors in TNBC patients. Encouraging results in a randomized phase II clinical trial demonstrated that the addition of iniparib, an oral PARP1 inhibitor, to gemcitabine and carboplatin improved clinical benefit rate, PFS and overall survival (OS) in metastatic TNBC patients compared with gemcitabine and carboplatin alone[20]. In another phase II trial of ABT888, also named as veliparib, which is an oral PARP1 and PARP2 inhibitor, the clinical response rate in BRCA1-mutation breast cancer patients was 37.5\% $(3 / 8)$, and there were no responses in non-BRCA1 carriers (0/33) (NCT01009788) [5] which was consistent with outcomes of another clinical trail [21]. The above results suggest that the role of PARP inhibitors in sporadic TNBC remain to be settled but it is explicit that they have the therapeutic potency in BRCA1-deficient breast cancers [22-24].

Besides the well-described functions in DDR, PARP1 also can function as a specific regulator for transcriptional functions of nuclear receptors. It has been reported that PARP1 can be recruited to AR function sites, and then promote AR occupancy and
AR functions. Data from multiple models showed that PARP1 triggered protumorigenic effects in AR-positive prostate cancer cells [25-27]. The effect of adding PARP inhibitor veliparib to androgen deprivation therapy has been explored in prostate cancer in clinical setting but the results are still unclear [26]. Intriguingly, BRCA1 is also reported to be a coactivator of AR and can increase expression of AR target genes [28, 29]. However, no researches have elucidated the relationship among BRCA1, PARP1 and AR in TNBC. And no systematic researches have studied the effect of combining androgen-blocking hormone treatment with PARP1 inhibitor in AR-positive TNBC. Our article is to further explore the correlation among AR, BRCA1 and PARP1 and evaluate the combination effect of bicalutamide and ABT-888 in AR-positive TNBC to explore new therapeutic approaches for TNBC patients.

\section{Materials and Methods}

\section{Reagents}

Bicalutamide and ABT- 888 with a purity of $99 \%$ were purchased from Selleck Chemicals. Dihydrotestosterone (DHT), 3-[4, 5-dimethylthiazol-2-yl]-2, 5-diphenyl tetrazolium bromide (MTT) and dimethyl sulfoxide (DMSO) were from Sigma Chemicals. DHT was dissolved in ethanol at $0.1 \mu \mathrm{M}$ concentration, bicalutamide and ABT- 888 were dissolved in DMSO at $10 \mu \mathrm{M}$ concentration. Cell Apoptosis Detection Kit was a product of KeyGEN Biotech, CA. The antibodies used included the following: AR (Abcam, ab74272), PARP1 (Abcam, ab32138). All culture media and serum were obtained from GIBCO/BRL Life Technologies, Inc.

\section{Cell lines and cell culture}

TNBC cell lines MDA-MB-231 and HCC1937 were obtained from the American Type Culture Collection (Manassas, VA, USA). Both of them were cultured in RPMI 1640 medium (GIBCO, Gaithersburg, MD, USA) supplemented with $10 \%$ fetal bovine serum (FBS) and $1 \%$ penicillin/streptomycin. Cell cultures were maintained at $37{ }^{\circ} \mathrm{C}$ with $5 \% \mathrm{CO} 2$ in a humidified atmosphere.

\section{Cell viability assay}

Human breast cancer cells were plated at a density of 10000 cells per well in flat-bottomed 96-well plates. The next day, cells were provided with fresh medium including $10 \mu \mathrm{M}$ bicalutamide and $10 \mu \mathrm{M}$ ABT-888 alone or in combination for $24 \mathrm{~h}, 48 \mathrm{~h}, 72 \mathrm{~h}$. After that the cells were incubated with $10 \mu \mathrm{l}$ of 0.5 $\mathrm{mg} / \mathrm{ml}$ MTT solution at $37^{\circ} \mathrm{C}$ for $4 \mathrm{~h}$. The resulting crystal was dissolved in $200 \mu \mathrm{l}$ DMSO per well, and 
the absorbance was measured with $490 \mathrm{~nm}$ with a microplate reader (BIO-RAD, USA).

\section{Colony formation assay}

MDA-MB-231 and HCC1937 were seeded in six-well plates at a density of 500 cells per well for approximately $24 \mathrm{~h}$ under standard conditions. After specific treatments, the cells then were exposed to bicalutamide and ABT-888 alone or in combination. After 10 days (MDA-MB-231) and 14 days (HCC1937) of incubation, the colonies were fixed with methanol, stained with $0.5 \%$ crystal violet in absolute ethanol, and colonies with $\geq 50$ cells were counted under a dissection microscope. These experiments were repeated at least three times.

\section{Flow cytometry analysis}

Human breast cancer cells were treated with bicalutamide and ABT-888 alone or in combination for $48 \mathrm{~h}$, then harvested by trypsinization (not with EDTA), centrifuged and washed with PBS. Cell pellets were resuspended and incubated in $500 \mu l$ binding buffer. After 15 minutes incubated with $5 \mu l$ annexin V-fluorescein isothiocyanate (FITC) and $5 \mu 1$ propidium iodide (PI) in the dark at room temperature, the rate of early apoptosis was monitored with a flow cytometer (BD FACS calibur, USA). Each sample was tested in triplicate.

\section{Plasmids and transient transfections}

Plasmid pBABEpuro HA BRCA1 (Plasmid \#14999) was purchased from Addgene (USA) and plasmid PARP1 was synthesized by Genepharma (Shanghai, China). MDA-MB-231 and HCC1937 cells were seeded at a density of $1 \times 106$ cells/plate in a 6-well plate with $10 \%$ FBS. After $24 \mathrm{~h}$, cells were transfected with a plasmid encoding BRCA1 sequence or the pBABE-puro as vehicle were performed in opti-MEM with the transfection reagent Lipofectamine $^{\mathrm{TM}} 2000$ (Invitrogen, CA, USA) following the manufacturer's instructions. After incubation at $37^{\circ} \mathrm{C}$ for $48 \mathrm{~h}$, cells were collected to measure by other analysis.

\section{Quantitative real-time polymerase chain reaction (qRT-PCR)}

Total RNA was extracted from cultured cells using Trizol Reagent (Invitrogen, Carlsbad,CA,USA) according to the manufacturer's instructions. For quantitative RTPCR analysis of BRCA1, AR and PARP1, $2 \mu \mathrm{g}$ total RNA was reverse transcribed to cDNA with oligdT primers and Thermoscript (TaKaRa, Dalian, China). Primer sequences (forward and reverse, respectively) were as follows: BRCA1, 5'-CCAAGGTTAGAGAGTTGGACAC-3' and 5'-GAAACCGTGCCAAAAGACTTC-3';
5'-GGGCGAAGTAGAGCATCCT-3' and 5'-GACGACCAGATGGCTGTCATT-3'; 5'-TTTCCATCAAACATGGGCGAC-3' 5'-CGGAGTCTTCGGATAAGCTCT-3'; 5'-AAGTGGTCGTTGAGGGCAATG-3' PARP1, and GAPDH, and 5'-CTGGGCTACACTGAGCACC-3'. Real-time PCR analyses of BRCA1, AR and PARP1 were performed on an ABI 7300 Sequence Detection System (Applied Biosystems, Foster City, CA, USA) using SYBR green dye (Invitrogen, Carlsbad, CA, USA). A $20 \mu$ reaction volume included $1 \mu \mathrm{l}$ cDNA, $1 \times$ QuantiTect SYBR green PCR Master Mix, and $0.5 \mu \mathrm{M}$ of sense and 0.5 $\mu \mathrm{M}$ of antisense primer. All PCRs were performed in triplicate. Threshold cycles (CT) were determined using fixed threshold settings.

\section{Western blot analysis}

Total protein was extracted using RIPA buffer supplemented with protease and phosphatase inhibitors and quantitied using a BCA kit (Thermo Scientific ,Pittsburgh PA,USA) and $20 \mu \mathrm{g}$ of protein was loaded in each lane and separated on a sodium dodecylsulfate-polyacrylamide (SDS-PAGE) gel and blotted onto nitrocellulose. Blots were blocked with $5 \%$ dry milk in tris-buffered saline/ $0.1 \%$ tween- 20 and incubated overnight with a diluted solution of primary antibody at $4^{\circ} \mathrm{C}$, followed by horseradish peroxidase-conjugated secondary antibody (1:5000) for $2 \mathrm{~h}$. The specific antibodies used for western blot were rabbit anti-AR antibody (1:200, ab74272) and were rabbit anti-PARP1 antibody (1:1000, ab32138). Bands were normalized to GAPDH expression, which was used as an internal loading control. Results from at least two separate experiments were analyzed.

\section{Immunohistochemistry staining}

Slides were deparaffinized in a series of xylenes and ethanols. Heat-mediated antigen retrieval was fulfilled with citrate buffer (BioGenex Laboratories, San Ramon, CA). Antibodies used were AR (1:50), PARP1 (1:25). Immunostained sections were scanned using a microscope at $\times 200$ magnification (Carl Zeiss, Germany). The total number of AR, PARP1and Ki-67 positive cells in a tumor cross-section was counted to measure in each sample.

\section{Mice xenograft models and immunohistochemistry analysis}

All animal experiments strictly followed the guidelines of the Institutional Review Board of Jinling Hospital. Approximately $5.0 \times 10^{6}$ cells were in $100 \mu \mathrm{l}$ PBS and injected subcutaneously into the right side of the posterior flank of female BALB/c athymic nude mice (Department of Comparative Medicine, Jinling Hospital, Nanjing, China) at 5 to 6 weeks of age. Tumor volumes were examined every other day and 
were calculated using the equation: $\mathrm{V}=\mathrm{A} \times$ $\mathrm{B}^{2} / 2\left(\mathrm{~cm}^{3}\right)$, where $\mathrm{A}$ is the largest diameter and $\mathrm{B}$ is the perpendicular diameter. When the average tumor size reached approximately $0.03 \mathrm{~cm}^{3}$, bicalutamide $(20 \mathrm{mg} / \mathrm{kg} / \mathrm{d})$ and ABT- $888(25 \mathrm{mg} / \mathrm{kg} / \mathrm{d})$ was administered via intraperitoneal injection every other day. After 3 weeks, all mice were killed, and necropsies were performed. Harvested tumors were fixed in formalin and embedded in paraffin for immunohistochemistry (IHC) staining.

\section{Statistical analysis}

SPSS Statistics 19.0 (SPSS Inc.) was used for statistical analysis. Data were analyzed using one-way ANOVA or a Student's t-test. Data are presented as means \pm the standard deviation (SD) of three independent experiments. All analyses were performed at a significance level of ${ }^{*} \mathrm{P}<0.05$ or $* * \mathrm{P}<0.001$.

\section{Results}

\section{Combination of bicalutamide and ABT-888 can inhibit cell viability and induce cell apoptosis in AR positive TNBC cells}

Many studies have indicated that $A R$ is associated with prognosis of breast cancer patients and influences endocrine treatment response in breast cancer, particularly in TNBC [30-33]. Our previous

A
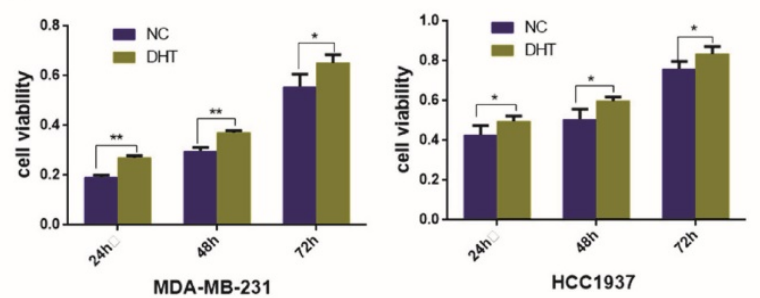

C
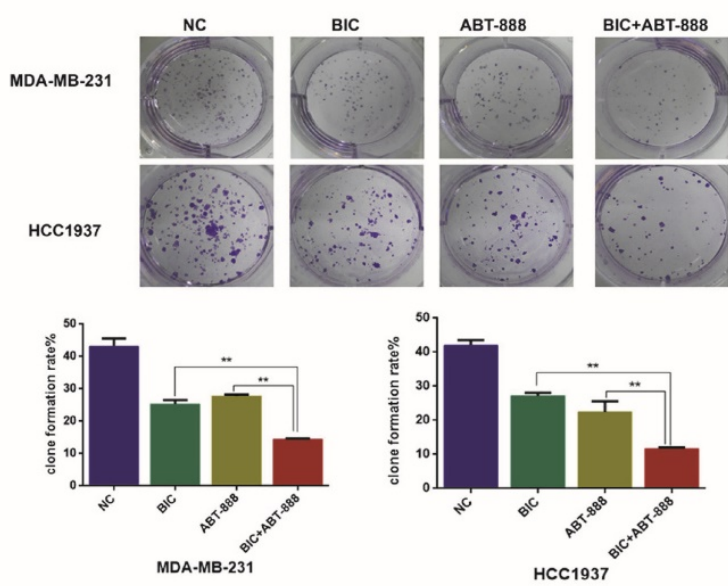

data showed that low AR expression was associated with better OS (103.18 vs. 84.71 months; $\mathrm{P}=0.002$ ) [34]. To investigate the effect of AR in the TNBC cell proliferation, we treated two AR-positive TNBC cell lines MDA-MB-231 and HCC1937 with AR agonist dihydrotestosterone (DHT), and then we detected obvious increase of cell viability in the two cell lines by MTT assay (Figure 1A).

PARP inhibition and BRCA1 mutation can result in synthetic lethality for breast cancer cells and as TNBC and BRCA1-mutated breast cancer share many characteristics, it is assumed that TNBC will be susceptible to PARP1 inhibitors [19]. In our study, we observed that ABT888 inhibited cell viability in MDA-MB-231 and HCC1937 cells significantly (Figure 1B). To demonstrate whether combination therapy of bicalutamide and ABT-888 make the growth inhibition stronger compared with monotherapy in TNBC cells, MDA-MB-231 and HCC1937 cell lines were treated with bicalutamide or ABT-888 alone or in combination. We observed that bicalutamide plus ABT-888 could reduce cell viability more significantly in contrast to bicalutamide or ABT-888 monotherapy (Figure 1B). These results were in accordance with clone formation rate (Figure 1C). In addition, combination therapy could induce cell apoptosis more significantly compared with bicalutamide or ABT-888 monotherapy (Figure 1D).

B
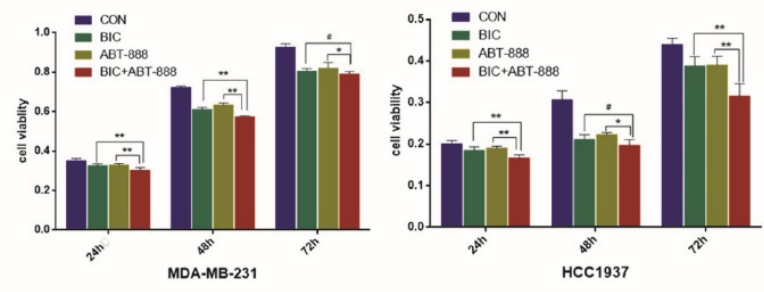

D
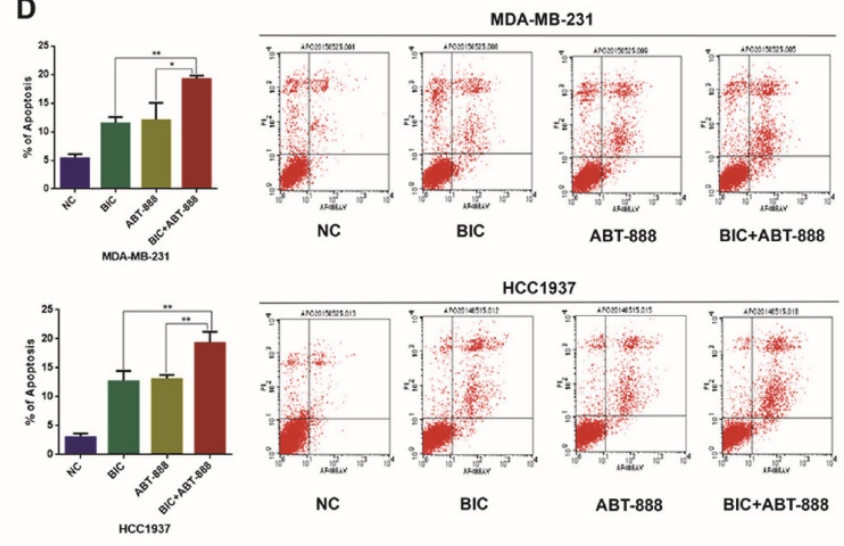

Figure 1. Combination of bicalutamide and ABT-888 can inhibit cell viability and induce cell apoptosis in AR positive TNBC cells. (A) MDA-MB-231 and $\mathrm{HCC} 1937$ cells were treated with $0.1 \mu \mathrm{M}$ DHT for $24 \mathrm{~h}, 48 \mathrm{~h}, 72 \mathrm{~h}$, and cell viability was determined by MTT assay. (B) MDA-MB-231 and HCC1937 cells were treated with $10 \mu \mathrm{M}$ bicalutamide and $10 \mu \mathrm{M}$ ABT-888 alone or in combination for $24 \mathrm{~h}, 48 \mathrm{~h}, 72 \mathrm{~h}$, and cell viability was determined by MTT assay. (C) MDA-MB-231 and HCC1937 cells were treated with $10 \mu \mathrm{M}$ bicalutamide and $10 \mu \mathrm{M}$ ABT-888 alone or in combination for $48 \mathrm{~h}$, then cell proliferation was determined by clone formation assay. (D) Cell apoptosis measured by

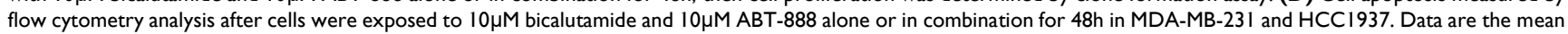
of three independent experiments. $* \mathrm{P}<0.05, * * \mathrm{P}<0.01, \# \mathrm{P}>0.05$. 

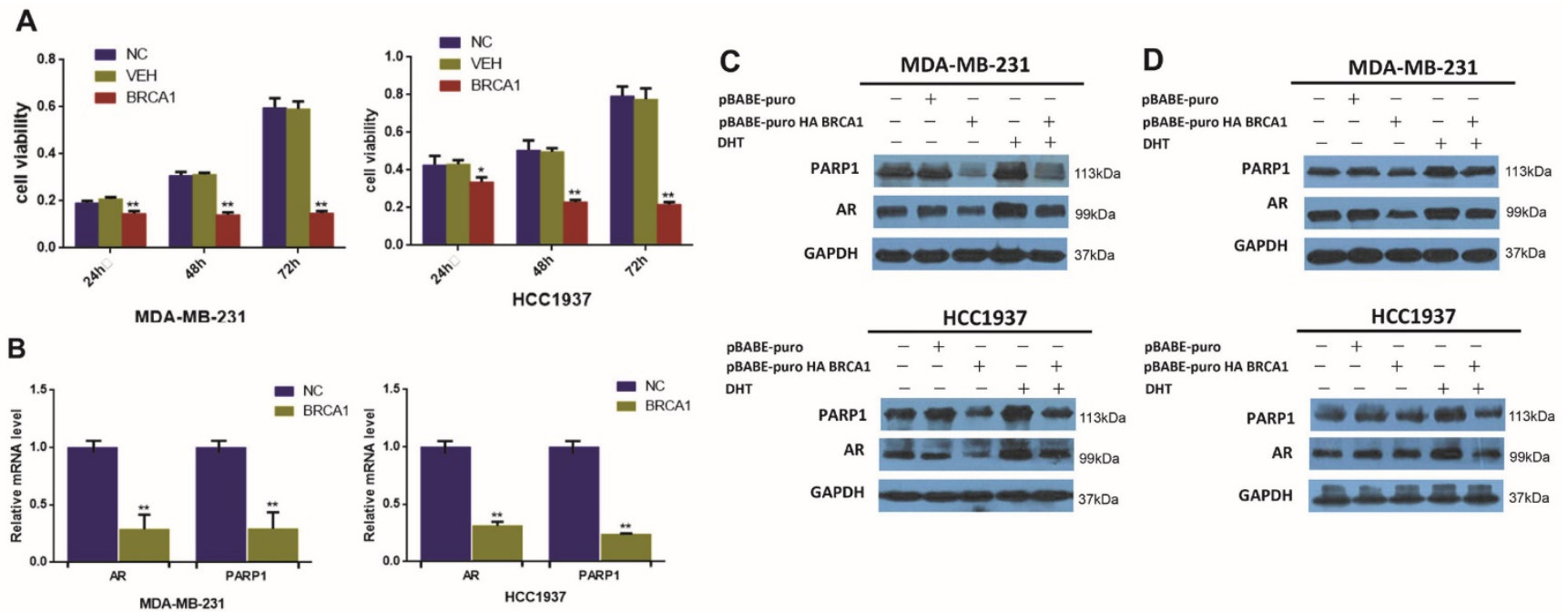

Figure 2. BRCA1 downregulates the expression of AR and PARP1 in AR-positive TNBC cells. (A) MDA-MB-231 and HCC1937 cells were transfected by plasmid encoding BRCA1 for $24 \mathrm{~h}, 48 \mathrm{~h}, 72 \mathrm{~h}$, and cell viability was determined by MTT assay. (B) AR and PARP1 mRNA levels were determined by RT-PCR following transfection of plasmid encoding BRCA1 for 24h in MDA-MB-231 and HCC1937, and normalized to GAPDH expression. (C) MDA-MB-231 and HCC1937 cells were transfected by plasmid encoding PBABE-puro as normal control and BRCA1, and treated with $0.1 \mu \mathrm{M} \mathrm{DHT}$ for $48 \mathrm{~h}$, and AR and PARPI protein levels were determined by western blot. (D) MDA-MB-231 and HCC1937 cells were transfected by plasmid encoding PBABE-puro as normal control, BRCA1, PARP1, or co-overexpression of BRCA1 and PARP1 for 48h, $\mathrm{AR}$ and PARPI protein levels were determined by western blot. $* \mathrm{P}<0.05, * * \mathrm{P}<0.01$

To conclude, these results suggested that AR might be associated with a poor prognosis in TNBC and combination of bicalutamide and ABT-888 could inhibit cell viability and induce cell apoptosis significantly in vitro.

\section{BRCAI negatively relates to AR and PARPI expression in vitro}

BRCA1 and PARP1 are both very important DNA damage response protein that promote DNA repair [35]. And interestingly, previous researches have found that both BRCA1 and PARP1 can interact with AR and influence the transcription of AR itself and its target genes in other cancer cells [25, 28]. However, little is known regarding the regulation effect of BRCA1 on AR and PARP1 in TNBC. We observed that BRCA1 overexpression could suppress cell viability in TNBC (Figure 2A). To further determinate the changes of AR and PARP1 induced by BRCA1 overexpression, we examined the levels of AR and PARP1 after over-expressing BRCA1 in TNBC cell lines. The relative mRNA levels of AR and PARP1 were significantly declined following BRCA1 overexpression in MDA-MB-231 and HCC1937 cells (Figure 2B). Decreased protein expression of AR and PARP1 were also detected in BRCA1-transfected TNBC cells (Figure 2C). In TNBC cells treated by DHT after overexpressing BRCA1, both AR and PAPR1 expressions were higher than the cells overexpressing BRCA1 and lower than the cells treated by DHT (Figure 2C). So, we proved that overexpression of BRCA1 could down regulate the expression of AR and PARP1 and we also assumed that the activation of AR by DHT might up regulate expression of
PARP1 in AR-positive TNBC cells. We then demonstrated the TNBC cells with co-overexpression of BRCA1 and PARP1 had higher expression of AR and PARP1 compared with TNBC cells overexpressing BRCA1 and lower expression of AR and PARP1 compared with TNBC overexpressing PARP1 (Figure 2D). The results once again demonstrated that overexpression of BRCA1 could down regulate the expression of AR and PARP1 and PARP1 might up-regulate expression of AR in AR-positive TNBC cells.

In conclusion, these results suggested that overexpression of BRCA1 could inhibit cell viability, and decrease AR and PARP1 expression in vitro.

\section{AR up-regulates PARPI expression, and PARPI also positively regulates $A R$ expression in vitro}

Based on the above results, we found that not only BRCA1 could decrease expression of both AR and PARP1, but also AR and PARP1 interacted with each other. And numerous researches on prostate cancer have revealed that antitumor response to PARP1 inhibition is associated with diminished AR activity [25]. Mechanistically, PARP1 is recruited to the binding sites of $A R$ and then promote $A R$ occupancy and AR transcriptional function [36]. However, the effect of AR on PARP1 has not been studied. So, we first detected the AR and PARP1 protein levels in AR-positive TNBC cells after activating AR by DHT or inhibiting AR by bicalutamide. And our data indicated that PARP1 expression was elevated by DHT and reduced by 
bicalutamide in contrast to control group (Figure 3A). These results were verified in mRNA levels measured by RT-PCR (Figure 3B). We concluded that AR activation could positively regulate PARP1 expression in TNBC cells.

To further explore whether PARP1 can affect AR expression in TNBC, we measured the protein level of AR in TNBC cells after transfection of PARP1 vector or treatment with ABT-888. Then we found that mRNA and protein expression levels of AR were increased by PARP1 overexpression and reduced by ABT-888 in AR-positive TNBC cells compared with the control group (Figure 3C, D). In conclusion, these data indicated that AR up-regulated PARP1, and PARP1 also up-regulated AR expression in vitro.

\section{Combination of bicalutamide and ABT-888 inhibits tumor growth in mice xenograft model}

To confirm our in vitro findings in vivo, we conducted nude mice xenograft models with HCC1937 cells and then randomly assigned them to one of four different treatment groups: control group, bicalutamide $(20 \mathrm{mg} / \mathrm{kg} / \mathrm{d})$ group, ABT- 888 $(25 \mathrm{mg} / \mathrm{kg} / \mathrm{d})$ group, biclutamide plus ABT-888 group. Over three weeks, our data indicated that co-treatment with bicalutamide and ABT-888 significantly delayed tumor growth compared with bicalutamide or ABT-888 single treatment (Figure 4C). We also observed that tumor tissues from the mice xenografts treated with bicalutamide plus ABT-888 showed lower Ki-67 expression compared to the other groups, suggesting combination treatment significantly reduced proliferation ability of tumor cells (Figure 4D). The result was corresponded with
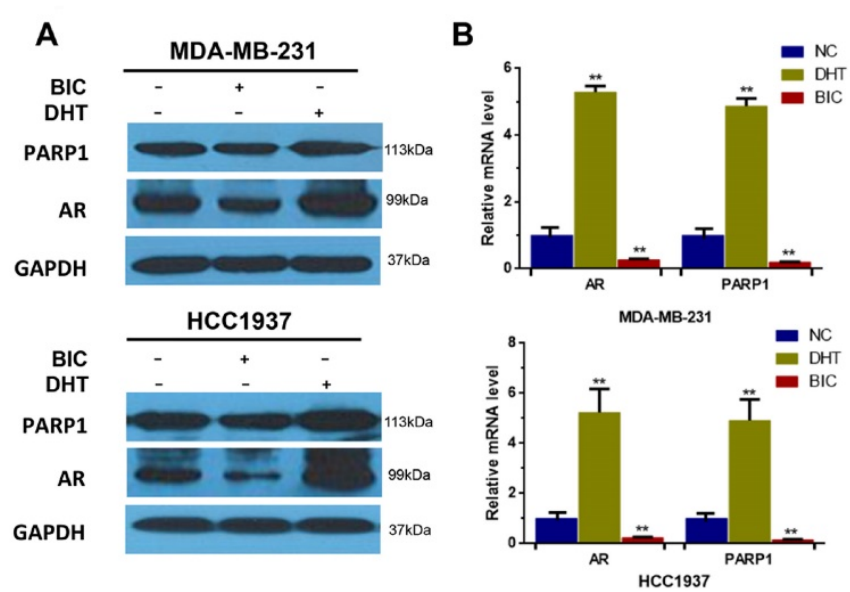

results of cell apoptosis by flow cytometer assay (Figure 1D). Additionally, the expression of AR and PARP1 were reduced more obviously in co-treatment group by using immunohistochemistry staining (Figure 4D). We could summarize that co-treatment with bicalutamide and ABT-888 significantly inhibited tumor growth in mice xenograft model.

\section{The inhibitory effect of combination of bicalutamid and ABT-888 on mice xenograft model is possibly associated with BRCAI status in TNBC}

Many researches have proved that PARP inhibition in BRCA1-mutated TNBC cell can induce synthetic lethality $[37,38]$. PARP inhibitor AG014699 delayed growth of xenograft tumor generated from TNBC cell MDA-MB-436 and HCC1937 which carried BRCA1 mutation more markedly in contrast with xenograft tumors with BRCA1/2-nonmutated breast cancer cells such as MCF-7, MDA-MB-231, and HCC1937-BRCA1 (derived from HCC1937 by correct the BRCA1 mutation)[39]. The previous study indicated that PARP inhibitor could increase sensibility of chemotherapy through DDR via BER pathway in BRCA1 mutation cancers [40]. We hypothesized that combination therapy of PARP1 inhibitor and bicalutamide could also increase anti-tumour growth effect more obviously in BRCA1-muated TNBC cells than BRCA-wild type cells. To examine the hypothesis in vivo, we established two xenograft models with BRCA1-mutation HCC1937 and BRCA1 wild-type MDA-MB-231 cells on either side of a nude mouse (Figure 5A). We assumed the two xenograft models were in the analogous microenvironment, and then

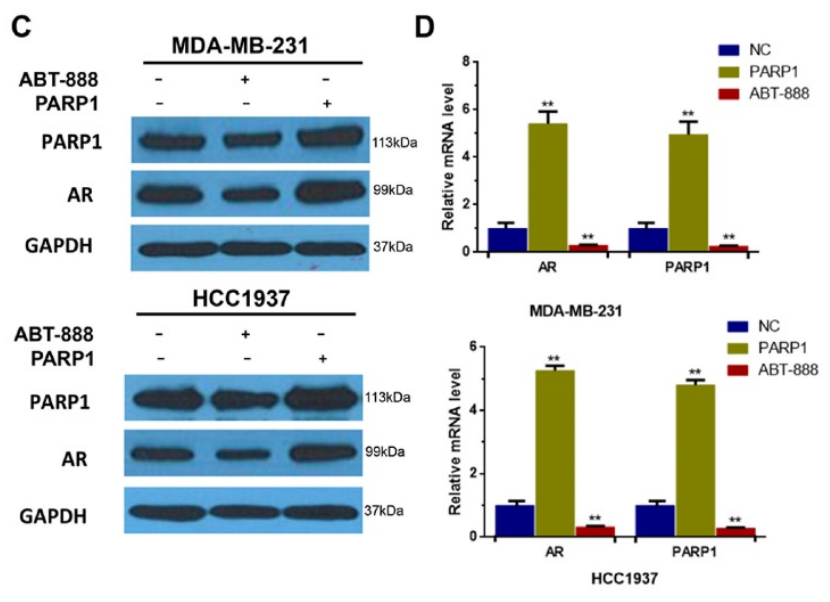

Figure 3. AR up-regulates the expression of PARPI and PARPI also positively regulates AR expression in AR-positive TNBC cells. (A) MDA-MB-231 and HCC1937 cells were treated with $10 \mu \mathrm{M}$ bicalutamide or $0.1 \mu \mathrm{M}$ DHT for $48 \mathrm{~h}$. The expression levels of AR and PARPI were determined by western blot, and normalized to

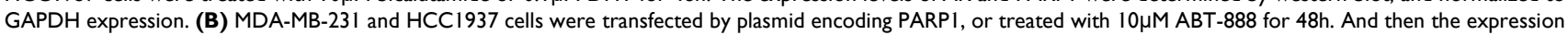
of AR and PARPI in these two groups were determined by western blot, and normalized to GAPDH expression. (C) MDA-MB-231 and HCC1937 cells were treated with $10 \mu \mathrm{M}$ bicalutamide or $0.1 \mu \mathrm{M}$ DHT for 24h. The mRNA levels of AR and PARP1 were determined by RT-PCR, and normalized to GAPDH expression. (D) MDA-MB-231 and HCC1937 cells were transfected by plasmid encoding PARP1, or $10 \mu \mathrm{M}$ ABT-888 for $24 \mathrm{~h}$. And then the mRNA levels of AR and PARP1 were determined by RT-PCR, and normalized to GAPDH expression. Data are the mean of three independent experiments. $* * \mathrm{P}<0.01$. 
treated the nude mouse with bicalutamide and ABT-888 by intraperitoneal injection for three weeks. We observed that the tumor volume growth was inhibited obviously in HCC1937 xenograft compared with MDA-MB-231 xenograft (Figure 5B). Collectively, the inhibitory effect of combination of bicalutamid and ABT-888 on tumor proliferation was possibly more obvious in BRCA1-mutated TNBC than BRCA1 wild-type TNBC.

Table 1. BRCAl expression was negative correlation with $A R$ and PARPI by IHC using the breast cancer tissue-array.

\begin{tabular}{|c|c|c|c|c|c|}
\hline & & Tumor microa & ray $(n=28)$ & & \\
\hline & & BRCA1(high) & BRCA1(low) & & \\
\hline & & $18 / 28(64.3 \%)$ & $10 / 28(35.7 \%)$ & $\begin{array}{l}P \\
\text { value }\end{array}$ & $\begin{array}{l}r \\
\text { value }\end{array}$ \\
\hline AR(high) & $8 / 28(28.6 \%)$ & 2 & 6 & & \\
\hline AR(low) & $20 / 28(71.4 \%)$ & 16 & 4 & 0.011 & -2.694 \\
\hline PARP1(high) & $14 / 28(50 \%)$ & 6 & 8 & & \\
\hline PARP1(low) & $14 / 28(50 \%)$ & 12 & 2 & 0.046 & -2.324 \\
\hline
\end{tabular}

A

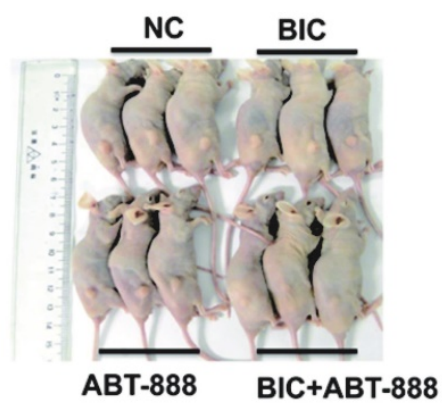

B

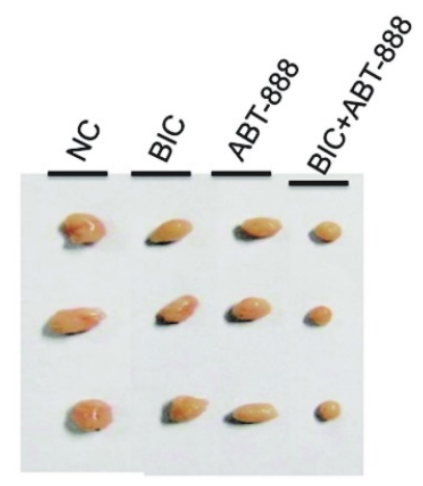

HCC1937

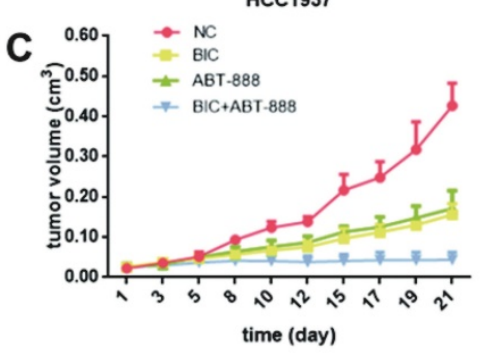

D

AR

NC

BIC
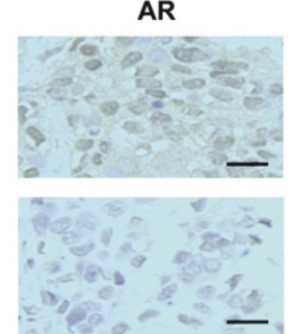

ABT-888

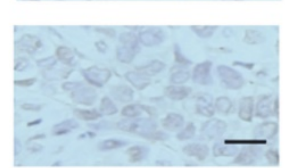

BIC+ABT-888

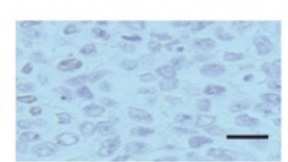

PARP1
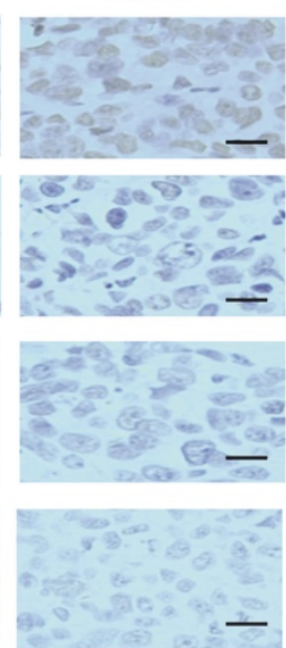

BRCA1, AR and PARP1 expression in triple-negative breast cancer patients

Based on these findings, we were interested in whether there was a connection among BRCA1, AR and PARP1 expression in TNBC patients, and whether the speculation that BRCA1 was negatively correlated with AR and PARP1 was correct. Therefore, we performed immunohistochemical staining of these proteins in malignant tumor samples from 28 TNBC patients using a tissue microarray. Representative staining is shown in Fig. 6A. We observed that 18 cases $(64.3 \%)$ and 10 cases (35.7\%) expressed BRCA1 at high and low levels, respectively (Table 1). We next analyzed the correlation among BRCA1, AR and PARP1 expression in the tumor tissues. An inverse correlation was identified between BRCA1 and AR expression ( $\mathrm{r}=-2.694, \mathrm{P}=0.011)$, which was consistent to our previous data [34]. Similarly, BRCA1 and PARP1 status also showed a significantly negative correlation $(\mathrm{r}=-2.324, \mathrm{P}=0.046)$ (Table 1). Therefore, BRCA1 expression was negative correlated with both $\mathrm{AR}$ and PARP1 in TNBC patients.

Figure 4. The inhibitory effects of combination of bicalutamide and ABT-888 in HCC1937 tumors growth in mice xenograft models. Mouse xenograft models with $\mathrm{HCCl} 937$ human breast cancer cells were established. Then we randomly assigned them to one of four different treatment groups: untreated group as normal control, bicalutamide $(20 \mathrm{mg} / \mathrm{kg} / \mathrm{d})$ group, ABT-888 $(25 \mathrm{mg} / \mathrm{kg} / \mathrm{d})$ group, and bicalutamide $(20 \mathrm{mg} / \mathrm{kg} / \mathrm{d})$ plus ABT-888 (25 mg/kg/d) group for 21 days. (A) There are the representative images of xenograft tumors after three weeks treatment. (B) The final tumors from the mouse xenograft models in the different four groups after 21 days are shown. (C) The growth curves of $\mathrm{HCCl} 937$ tumors growth in mice xenograft models, representing mean volumes of 3 subcutaneous transplanted tumors per group, measured twice days with calipers, are presented in the graph with the standard deviation. (D) The tumors were removed from the mice in 15 days after drug treatment ended, and immunohistochemical staining for AR, PARPI, and Ki-67 were conducted. Photomicrographs depict representative AR, PARPI, and Ki-67 staining (200× magnification). 
A

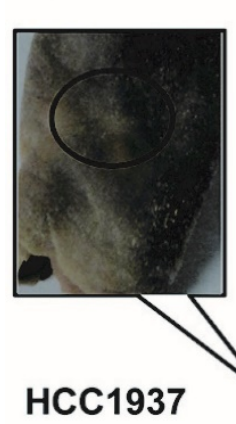

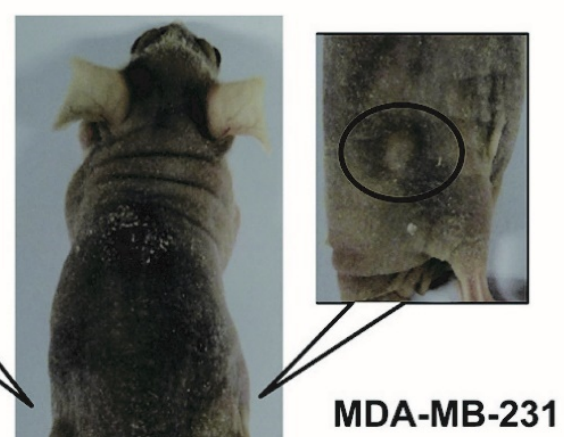

MDA-MB-231

\section{B}

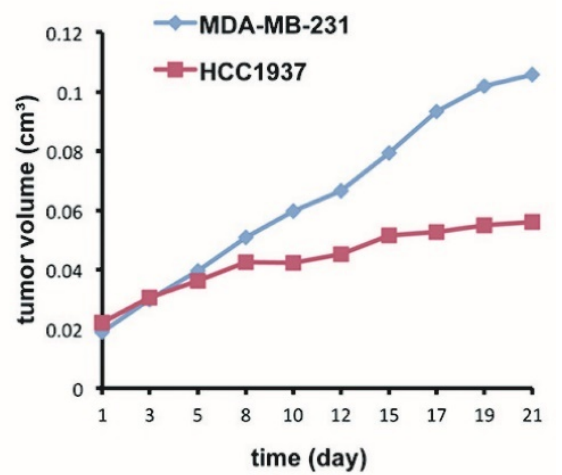

Figure 5. The inhibitory effect of combination ABT-888 and bicalutamide on mice xenograft model growth effect is possibly associated with BRCA1 status in TNBC. (A) We conducted two xenograft models with HCC1937 and MDA-MB-231 cells in left and right sides of hind legs respectively of a nude mouse and treat the mouse with bicalutamide $(20 \mathrm{mg} / \mathrm{kg} / \mathrm{d})$ plus ABT- $888(25 \mathrm{mg} / \mathrm{kg} / \mathrm{d})$ for 3 weeks. (B) Fold change of each tumor volume measured twice days with calipers is shown in a graph.

A BRCA1
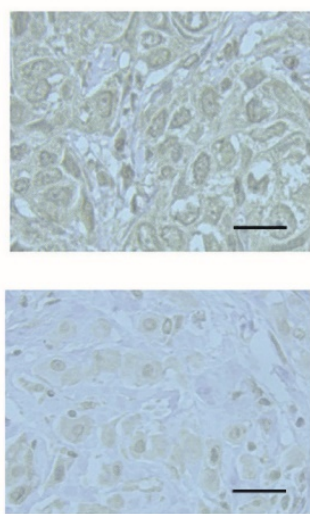

AR
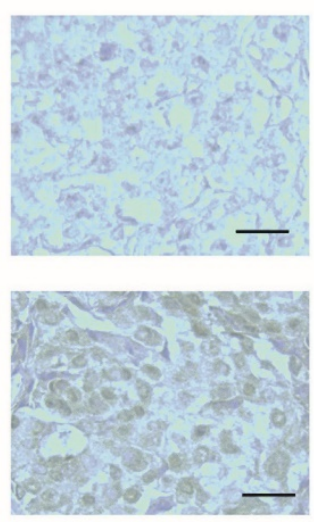

PARP1
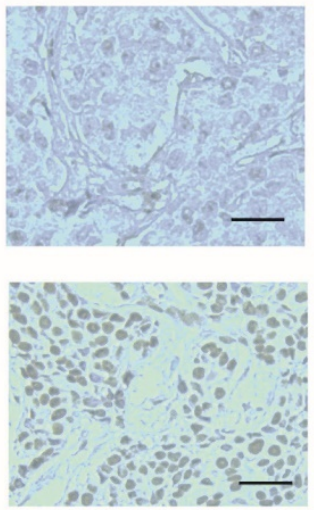

B

\section{BRCA1}

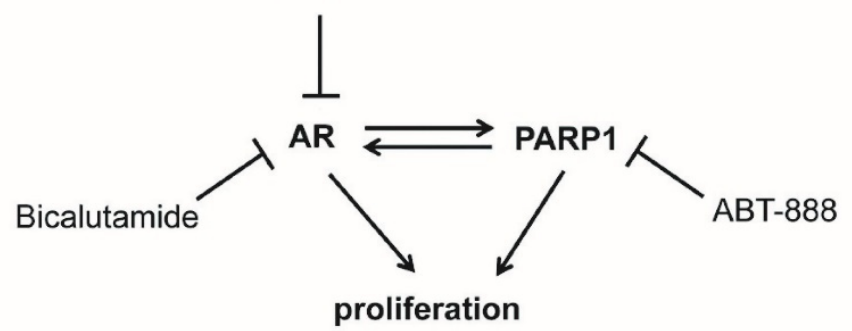

Figure 6. High BRCA1 expression is associated with low AR and PARPI expression in TNBC patients. (A) Immunohistochemical analysis of BRCAI, AR and PARP1 in TNBC patients by high magnification $(200 \times)$ is shown. (B) Schematic model depicts the relative effects of BRCA1, AR and PARP1 in regulatory pathway. 


\section{Discussion}

For lack of ER and PR expression, as well as HER-2 amplification, TNBC patients cannot benefit from conventional hormonal or anti-HER2 targeted therapies. It is delight that emerging researches have shown AR may be a therapy target for TNBC $[30,41]$. Here, we showed that activation of AR by DHT could promote cell proliferation and conversely inhibition of AR by bicalutamid could inhibit cell proliferation and induce cell apoptosis in AR-positive TNBC. AR is a member of nuclear receptors and is involved in cell cycle regulation and cell proliferation. In the absence of DHT, AR is located in the cytoplasm and forms a complex with heat shock protein [42]. Once AR binds with DHT in a ligand-dependent manner, AR homodimer will translocate to nuclear and interact with androgen responsive elements to regulate target gene transcription. In another ligand-independent manner without DHT, MAPK and STAT3 signal pathways can promote AR to translocate into nuclear [43]. AR knockdown can change cell cycle procession by distinctly down regulating of cyclin D1, D2, B and $\mathrm{A}$, and up-regulating p27 [44]. It is also validated that mRNA levels of EGF, SHC, PKC, GRB2 and RASA are significantly reduced in the presence of $A R$ knockdown, and increased after AR overexpression in another study [45]. A study clarified that bicalutamide treatment induced cell death in AR-positive TNBC cell MDA-MB-453 through directly inhibiting transcription of WNT7B and HER3 and then impairing WNT or HER2 signaling [46]. Our recent study found that AR-stimulated proliferation in breast cancer was associated with mTOR activity, in accordance to the findings in other cancer cells [47].

PARP1 is indispensable in BER pathway which repairs DNA single strand breaks and BRCA1-mutated TNBC patients should be sensitive to PARP1 inhibitors due to synthetic lethality [35]. Other than function involved in DDR, PARP1 has been reported to bind to gene promoters and to interact with transcriptional factors to mediate gene transcription. Based on roles for PARP1 in ER-a transcriptional activation, neuron-derived orphan receptor 1 and AR transcriptional regulations, it is assumed that PARP1 has a potential function in modulating transcriptional function of nuclear receptor and especially in hormone-dependent cancers [25]. As for modulation of AR transcriptional functions, it has been elucidated that PARP1 is recruited to the function sites of $A R$ and then promote AR-mediated target gene transcription including AR itself by enhancer binding, interaction with insulators, modulation of chromatin structure, and/or regulation of transcription factors [25]. Emerging researches have suggested that PARP1 inhibitors can disrupt transcriptional activities of AR and suppress AR-dependent cellular proliferation both in vitro and in vivo in prostate cancer [25, 26]. Although PARP1-mediated transcriptional effects have been studied much in prostate cancer, the impact of the transcriptional function of PARP1 in TNBC has never been delineated. In our data, inhibition of PARP1 by ABT-888 could increase cell death and inhibit the tumor growth in mice xenograft models. And we assumed that suppression of cell viability from PARP-1 inhibitor in AR-positive TNBC was due to not only impaired DNA damage repair but also reduced AR activity. We confirmed that PARP1 could also up regulate AR and interestingly AR positively regulated PARP1 in this paper. However, the mechanism of AR up regulating PARP1 still needs to be further studied.

The role of BRCA1 in ligand-dependent AR signaling has been studied for more than a decade. In previous studies, BRCA1 could regulate AR through binding to the AR AF-1 site to inhibit AR transcriptional function and BRCA1 could also inhibit AR-mediated proliferation of breast cancer cells through the activation of SIRT1[48, 49]. In our study, it was found that BRCA1 could down regulate the expression of AR and PARP1. We assumed that the PARP1 down-regulation after BRCA1 overexpression might be associated with the inhibition of AR.

Besides the indirect relationship between BRCA1 and PARP1 due to the BRCA-PARP synthetic lethality, direct interactions between these two proteins also exist. BRCA1 inactivation due to mutation or promoter methylation is followed with increased PARP1 and nicotinamide adenine dinucleotide (NAD) levels and interestingly the high efficiency of PARP1 induced by BRCA1 inactivation may further inhibit BRCA1 transcription by NAD depletion. Besides, recent studies have found that PARP1 also participate in BRCA1-mediated HRR. PARP1 is observed to interact with and poly-ADP-ribosylates (aka PARsylates) BRCA1. PARsylation is directed at the BRCA1 DNA binding domain and reduce its function to avoid excessive HRR and maintain genome stability [50]. In this study, we found that BRCA1 expression influenced the PARP1.These evidences emphasize that interaction between BRCA1 and PARP1 may be beneficial for the dynamic balance between BRCA1 and PARP1- associated biologic processes.

We reported strengthened effect of inhibiting cell viability and inducing cell apoptosis by combining AR antagonist bicalutamide with PARP inhibitor ABT-888 in AR positive TNBC cell lines. Importantly, combination of bicalutamide and ABT-888 could significantly inhibit tumor growth in mice xenograft 
model compared with monotherapy. We hope it will provide a new therapy strategy in AR-positive TNBC. And the study on relationship among BRCA1, PARP1 and AR will provide theories for more possible therapy strategies.

\section{Acknowledgements}

This research was supported by the Research Innovation Program for College Graduates of Jiangsu Province (Grant No. KYLX15_0048), National Natural Science Foundation of China (No.81470357), and Foundation for Clinical Medicine Science and Technology Special Project of the Jiangsu Province, China (No. BL2014071).

\section{Conflict of Interest} interest.

The authors declare no competing financial

\section{References}

1. Lehmann BD, Bauer JA, Chen X, Sanders ME, Chakravarthy AB, Shyr Y, et al. Identification of human triple-negative breast cancer subtypes and preclinical models for selection of targeted therapies. J Clin Invest. 2011; 121: 2750-67.

2. Dent R, Trudeau M, Pritchard KI, Hanna WM, Kahn HK, Sawka CA, et al. Triple-negative breast cancer: clinical features and patterns of recurrence. Clin Cancer Res. 2007; 13: 4429-34

3. Gucalp A, Traina TA. Triple-negative breast cancer: role of the androgen receptor. Cancer J. 2010; 16: 62-5.

4. Gasparini P, Fassan M, Cascione L, Guler G, Balci S, Irkkan C, et al. Androgen receptor status is a prognostic marker in non-basal triple negative breast cancers and determines novel therapeutic options. PLoS One. 2014; 9: e88525.

5. Lehmann BD, Pietenpol JA, Tan AR. Triple-negative breast cancer: molecular subtypes and new targets for therapy. Am Soc Clin Oncol Educ Book. 2015; 35: e31-9.

6. Choi JE, Kang SH, Lee SJ, Bae YK. Androgen receptor expression predicts decreased survival in early stage triple-negative breast cancer. Ann Surg Oncol. 2015; 22: 82-9.

7. Masiello D, Cheng S, Bubley GJ, Lu ML, Balk SP. Bicalutamide functions as an androgen receptor antagonist by assembly of a transcriptionally inactive receptor. J Biol Chem. 2002; 277: 26321-6.

8. Gucalp A, Tolaney S, Isakoff SJ, Ingle JN, Liu MC, Carey LA, et al. Phase II trial of bicalutamide in patients with androgen receptor-positive, estrogen receptor-negative metastatic Breast Cancer. Clin Cancer Res. 2013; 19: 5505-12.

9. Dantzer F, Nasheuer HP, Vonesch JL, de Murcia G, Menissier-de Murcia J. Functional association of poly(ADP-ribose) polymerase with DNA polymerase alpha-primase complex: a link between DNA strand break detection and DNA replication. Nucleic Acids Res. 1998; 26: 1891-8.

10. Oei SL, Herzog H, Hirsch-Kauffmann M, Schneider R, Auer B, Schweiger M. Transcriptional regulation and autoregulation of the human gene for ADP-ribosyltransferase. Mol Cell Biochem. 1994; 138: 99-104.

11. Wacker DA, Ruhl DD, Balagamwala EH, Hope KM, Zhang T, Kraus WL. The DNA binding and catalytic domains of poly(ADP-ribose) polymerase 1 cooperate in the regulation of chromatin structure and transcription. Mol Cell Biol. 2007; 27: 7475-85

12. Hassa PO, Hottiger MO. The diverse biological roles of mammalian PARPS, a small but powerful family of poly-ADP-ribose polymerases. Front Biosci. 2008; 13: 3046-82.

13. Koh DW, Dawson TM, Dawson VL. Mediation of cell death by poly(ADP-ribose) polymerase-1. Pharmacol Res. 2005; 52: 5-14.

14. Kolthur-Seetharam U, Dantzer F, McBurney MW, de Murcia G, Sassone-Corsi P. Control of AIF-mediated cell death by the functional interplay of SIRT1 and PARP-1 in response to DNA damage. Cell Cycle. 2006; 5: 873-7.

15. Gibson BA, Kraus WL. New insights into the molecular and cellular functions of poly(ADP-ribose) and PARPs. Nat Rev Mol Cell Biol. 2012; 13: 411-24.

16. Hartman AR, Kaldate RR, Sailer LM, Painter L, Grier CE, Endsley RR, et al. Prevalence of BRCA mutations in an unselected population of triple-negative breast cancer. Cancer. 2012; 118: 2787-95.

17. Gonzalez-Angulo AM, Timms KM, Liu S, Chen H, Litton JK, Potter J, et al. Incidence and outcome of BRCA mutations in unselected patients with triple receptor-negative breast cancer. Clin Cancer Res. 2011; 17: 1082-9.

18. Helleday T, Bryant HE, Schultz N. Poly(ADP-ribose) polymerase (PARP-1) in homologous recombination and as a target for cancer therapy. Cell Cycle. 2005; 4: 1176-8.
19. Farmer $\mathrm{H}$, McCabe N, Lord CJ, Tutt AN, Johnson DA, Richardson TB, et al. Targeting the DNA repair defect in BRCA mutant cells as a therapeutic strategy. Nature. 2005; 434: 917-21.

20. O'Shaughnessy J, Osborne C, Pippen JE, Yoffe M, Patt D, Rocha C, et al Iniparib plus chemotherapy in metastatic triple-negative breast cancer. New England Journal of Medicine. 2011; 364: 205-14.

21. Gelmon KA, Tischkowitz M, Mackay H, Swenerton K, Robidoux A, Tonkin K, et al. Olaparib in patients with recurrent high-grade serous or poorly differentiated ovarian carcinoma or triple-negative breast cancer: a phase 2, multicentre, open-label, non-randomised study. Lancet Oncol. 2011; 12: 852-61.

22. Tutt A, Robson M, Garber JE, Domchek SM, Audeh MW, Weitzel JN, et al. Oral poly(ADP-ribose) polymerase inhibitor olaparib in patients with BRCA1 or BRCA2 mutations and advanced breast cancer: a proof-of-concept trial. Lancet. 2010; 376: 235-44.

23. O'Shaughnessy J, Osborne C, Pippen JE, Yoffe M, Patt D, Rocha C, et al. Iniparib plus chemotherapy in metastatic triple-negative breast cancer. $\mathrm{N}$ Engl J Med. 2011; 364: 205-14.

24. Donawho CK, Luo Y, Luo Y, Penning TD, Bauch JL, Bouska JJ, et al. ABT-888, an orally active poly(ADP-ribose) polymerase inhibitor that potentiates DNA-damaging agents in preclinical tumor models. Clin Cancer Res. 2007; 13: 2728-37.

25. Schiewer MJ, Goodwin JF, Han S, Brenner JC, Augello MA, Dean JL, et al. Dual roles of PARP-1 promote cancer growth and progression. Cancer discovery. 2012; 2: 1134-49.

26. Zhang J. Poly (ADP-ribose) polymerase inhibitor: an evolving paradigm in the treatment of prostate cancer. Asian journal of andrology. 2014; 16: 401-6.

27. Pu H, Horbinski C, Hensley PJ, Matuszak EA, Atkinson T, Kyprianou N. PARP-1 regulates epithelial-mesenchymal transition (EMT) in prostate tumorigenesis. Carcinogenesis. 2014; 35: 2592-601.

28. Yeh $\mathrm{S}, \mathrm{Hu}$ YC, Rahman $\mathrm{M}$, Lin HK, Hsu CL, Ting HJ, et al. Increase of androgen-induced cell death and androgen receptor transactivation by BRCA1 in prostate cancer cells. Proc Natl Acad Sci U S A. 2000; 97: 11256-61.

29. Park JJ, Irvine RA, Buchanan G, Koh SS, Park JM, Tilley WD, et al. Breast cancer susceptibility gene 1 (BRCA1) is a coactivator of the androgen receptor. Cancer research. 2000; 60: 5946-9.

30. Ricciardi GR, Adamo B, Ieni A, Licata L, Cardia R, Ferraro G, et al. Androgen Receptor (AR), E-Cadherin, and Ki-67 as Emerging Targets and Novel Prognostic Markers in Triple-Negative Breast Cancer (TNBC) Patients. PLoS One. 2015; 10: e0128368.

31. Kwiatkowska E, Teresiak M, Filas V, Karczewska A, Breborowicz D, Mackiewicz A. BRCA2 mutations and androgen receptor expression as independent predictors of outcome of male breast cancer patients. Clin Cancer Res. 2003; 9: 4452-9.

32. Vera-Badillo FE, Templeton AJ, de Gouveia P, Diaz-Padilla I, Bedard PL, Al-Mubarak M, et al. Androgen receptor expression and outcomes in early breast cancer: a systematic review and meta-analysis. J Natl Cancer Inst. 2014; 106: djt319.

33. Kim Y, Jae E, Yoon M. Influence of Androgen Receptor Expression on the Survival Outcomes in Breast Cancer: A Meta-Analysis. J Breast Cancer. 2015; 18: 134-42.

34. Zhang W, Luo J, Yang F, Wang Y, Yin Y, Strom A, et al. BRCA1 inhibits AR-mediated proliferation of breast cancer cells through the activation of SIRT1. Sci Rep. 2016; 6: 22034.

35. Bouwman $\mathrm{P}$, Jonkers J. The effects of deregulated DNA damage signalling on cancer chemotherapy response and resistance. Nature Reviews Cancer. 2012; 12: $587-98$

36. Schiewer MJ, Goodwin JF, Han S, Brenner JC, Augello MA, Dean JL, et al. Dual roles of PARP-1 promote cancer growth and progression. Cancer Discov. 2012; 2: $1134-49$

37. Anders CK, Winer EP, Ford JM, Dent R, Silver DP, Sledge GW, et al. Poly(ADP-Ribose) polymerase inhibition: "targeted" therapy for triple-negative breast cancer. Clin Cancer Res. 2010; 16: 4702-10.

38. De P, Sun Y, Carlson JH, Friedman LS, Leyland-Jones BR, Dey N. Doubling down on the PI3K-AKT-mTOR pathway enhances the antitumor efficacy of PARP inhibitor in triple negative breast cancer model beyond BRCA-ness. Neoplasia. 2014; 16: 43-W19.

39. Drew Y, Mulligan EA, Vong W-T, Thomas HD, Kahn S, Kyle S, et al. Therapeutic potential of poly (ADP-ribose) polymerase inhibitor AG014699 in human cancers with mutated or methylated BRCA1 or BRCA2. Journal of the National Cancer Institute. 2011; 103: 334-46.

40. Oza AM, Cibula D, Benzaquen AO, Poole C, Mathijssen RHJ, Sonke GS, et al. Olaparib combined with chemotherapy for recurrent platinum-sensitive ovarian cancer: a randomised phase 2 trial. The Lancet Oncology. 2015; 16: 87-97.

41. Safarpour D, Pakneshan S, Tavassoli FA. Androgen receptor (AR) expression in 400 breast carcinomas: is routine AR assessment justified? Am J Cancer Res. 2014; 4: 353-68.

42. Zoubeidi A, Zardan A, Beraldi E Fazli L Sowery $R$, Rennie $P$ et al Cooperative interactions between androgen receptor (AR) and heat-shock protein 27 facilitate AR transcriptional activity. Cancer Res. 2007; 67: 10455-65.

43. Ueda T, Bruchovsky N, Sadar MD. Activation of the androgen receptor N-terminal domain by interleukin-6 via MAPK and STAT3 signal transduction pathways. J Biol Chem. 2002; 277: 7076-85 
44. Liu L, Li Y, Xie N, Shynlova O, Challis JR, Slater D, et al. Proliferative action of the androgen receptor in human uterine myometrial cells--a key regulator for myometrium phenotype programming. J Clin Endocrinol Metab. 2013; 98: 218-27.

45. Li H, Li Y, Morin D, Plymate S, Lye S, Dong X. The androgen receptor mediates antiapoptotic function in myometrial cells. Cell Death Dis. 2014; 5: e1338.

46. Ni M, Chen Y, Lim E, Wimberly H, Bailey ST, Imai Y, et al. Targeting androgen receptor in estrogen receptor-negative breast cancer. Cancer Cell. 2011; 20: 119-31.

47. Recchia AG, Musti AM, Lanzino M, Panno ML, Turano E, Zumpano R, et al. A cross-talk between the androgen receptor and the epidermal growth factor receptor leads to p38MAPK-dependent activation of mTOR and cyclinD1 expression in prostate and lung cancer cells. Int J Biochem Cell Biol. 2009; 41: 603-14.

48. Park JJ, Irvine RA, Buchanan G, Koh SS, Park JM, Tilley WD, et al. Breast cancer susceptibility gene 1 (BRCAI) is a coactivator of the androgen receptor. Cancer Res. 2000; 60: 5946-9.

49. Zhang W, Luo J, Yang F, Wang Y, Yin Y, Strom A, et al. BRCA1 inhibits AR-mediated proliferation of breast cancer cells through the activation of SIRT1. Scientific reports. 2016; 6: 22034.

50. Hu Y, Petit SA, Ficarro SB, Toomire KJ, Xie A, Lim E, et al. PARP1-Driven Poly-ADP-Ribosylation Regulates BRCA1 Function in Homologous Recombination-Mediated DNA Repair. Cancer discovery. 2014; 4: 1430-47. 